\title{
Oppositions in Rough Set Theory ${ }^{\star}$
}

\author{
Davide Ciucci $^{1,2 \star \star}$, Didier Dubois ${ }^{1}$, and Henri Prade ${ }^{1}$ \\ 1 IRIT, Université Paul Sabatier \\ 118 route de Narbonne, 31062 Toulouse cedex 9 (France) \\ 2 DISCo - Università di Milano - Bicocca \\ Viale Sarca 336 - U14, 20126 Milano (Italia)
}

\begin{abstract}
The role of opposition in rough set theory is laid bare. There are two sources which generate oppositions in rough sets: approximations and relations. In the former case, we outline a hexagon and a cube of oppositions. In the second case, we define a classical square of oppositions and also a tetrahedron when considering the standpoint of two agents.
\end{abstract}

\section{Introduction}

Starting from the Greek philosophy, in particular Aristotle, oppositions have been organized in a so-called square of opposition where each vertex represents a different statement involving two entities $\mathrm{X}$ and $\mathrm{Y}$ : "Every $\mathrm{X}$ is $\mathrm{Y}$ " (A); "Some $\mathrm{X}$ is not $\mathrm{Y}$ " $(\mathrm{O})$; "Every $\mathrm{X}$ is not $\mathrm{Y}$ " (E) and "Some $\mathrm{X}$ is Y" (I). Clearly, (A) and (I) are in opposition to (O) and (E) (and vice-versa), (A) implies (I) and (E) implies $(\mathrm{O})$. (A) and (E) can be false together but not true, and for $(\mathrm{I})$ and $(\mathrm{O})$ it is the converse. This organization of oppositions have been discussed by several authors until the Middle Ages, then it progressively lost its importance in logic. Only since the 1950s, we can observe a renewed interest on this topic. In particular, in the last years, several authors tried to generalize the notion of square to describe more complex situations and to apply the square and other geometrical organizations of oppositions to several fields $[2,3]$. We just mention the link with Belnap and paraconsistent logic, fixed-point calculus, possibility theory, formal concept analysis [1]. Here, we lay bare the links between several forms of opposition and rough sets: cube and hexagon of opposition naturally arise by lower and upper approximations and square and tetrahedron of oppositions are generated by relations. The aim is to give a new theoretical approach to rough sets with possible new links (and differences) to other paradigms such as formal concept analysis and possibility theory. New ideas about approximations with two relations/agents are also proposed.

\section{Preliminary Notions}

We now give the introductory notions about the geometrical organization of oppositions and rough set theory.

\footnotetext{
* Appeared in LNCS 7414/2012, 504-513, DOI: 10.1007/978-3-642-31900-6_62

** Supported by FP7-Marie Curie Action (IEF) n.276158.
} 


\subsection{Oppositions and Geometrical Organization}

The Aristotelian square of opposition described in the introduction can be generalized in two directions: either by negation of $\mathrm{X}$ and $\mathrm{Y}$, or by considering (U), the disjunction of $(\mathrm{A})$ and $(\mathrm{E})$, and $(\mathrm{Y})$ the conjunction of $(\mathrm{I})$ and $(\mathrm{O})$.

In the first case we obtain a cube of oppositions, where, besides the typical square statements we have: "Every (not $\mathrm{X})$ is $($ not $\mathrm{Y})$ " (a); "Some (not $\mathrm{X})$ is Y" (o); "Every (not X) is Y" (e) and "Some (not X) is (not Y)" (i).
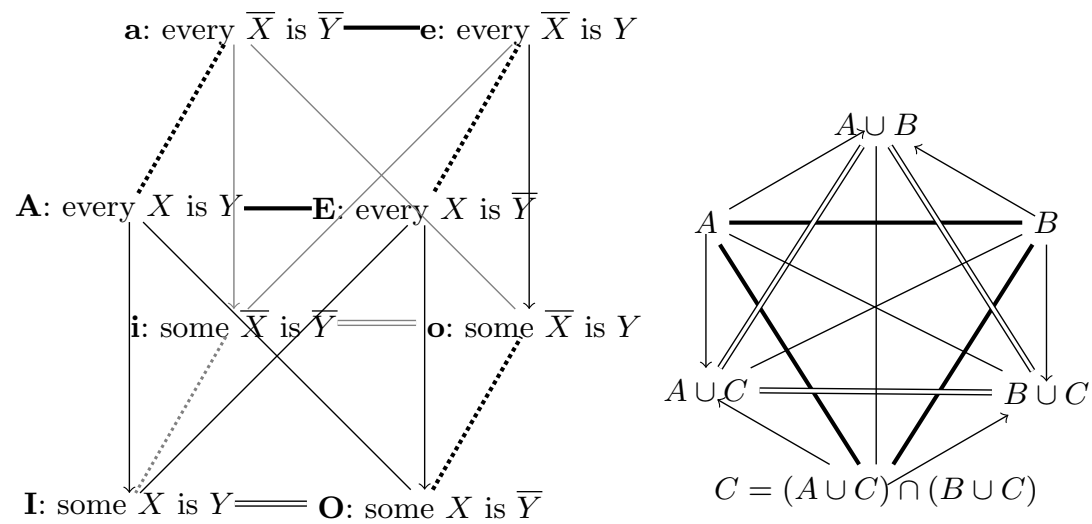

Fig. 1. Cube and hexagon of opposition

So, in the cube we have two squares of opposition: one in the front and the other in the back facet. In figure 1(left), the thick segment relates the contraries, the double thin undirected segments the sub-contraries, the diagonal non-directed segments the contradictories, and the vertical uni-directed segments point to subalterns (and express entailments if the set of $X$ 's and the set of $\bar{X}$ 's are not empty).

Example 2.1. Let $X=$ apple and $Y=$ red. Then, we have A: every apple is red; $\mathbf{E}$ : every apple is not red; $\mathbf{I}$ : some apple is red; $\mathbf{O}$ : some apple is not red; a: anything that is not an apple is not red; e: anything that is not an apple is red; i: something that is not an apple is not red; o: something that is not an apple is red.

In the second case, we obtain the hexagon of opposition proposed by Blanché as the starting point in his analysis of oppositions [4]. Hexagons naturally arise in different context, for instance in comparative relations in mathematics: (A): $=">",(\mathrm{E}):="<",(\mathrm{U}):=" \neq ",(\mathrm{I}):=" \geq ",(\mathrm{O}):=" \leq "$, and $(\mathrm{Y}):$ $="="$ or with tastes: $(\mathrm{A})=$ "I like it", $(\mathrm{E}):=$ "I dislike it", $(\mathrm{U}):=$ "I am not indifferent", (I): = "I do not dislike it", $(\mathrm{O}):=$ "I do not like it", and $(\mathrm{Y}):=$ "I am indifferent". Basically, we encounter an hexagon when we have a partition in three mutually exclusive situations [9]. Indeed, once considered a 3-partition 
of a universe of objects $O b j=A \cup B \cup C$, we obtain the hexagon of figure 1 (right). In this figure, thick segments link contraries and the double segments link sub-contraries.

As a further step, we can consider a 4-partition, i.e. a partition of a universe in four mutually exclusive subsets. In this case we obtain a tetrahedron of oppositions. An important example of tetrahedron is obtained by all the combinations of two Boolean statements $p, q$. Figure 2 gives a rich view of the relations among the binary connectives. We notice indeed that:

- any connective that appears between two others on the same segment (which may be an edge of the tetrahedron, or a segment linking a vertex to the middle of an opposite edge) is implied by each of the two connectives at the extremities;

- contradiction and tautology can both be associated with the center of the tetrahedron. Indeed, a segment linking the middle of two opposite edges, or a segment linking a vertex to the center of the opposite facet, is associated with a pair of connectives (at its extremities) whose conjunction yields the contradiction, and their disjunction yields the tautology.

- the position of a connective on a segment reflects the number of bits to switch for moving from it to the connectives that are at its extremities.

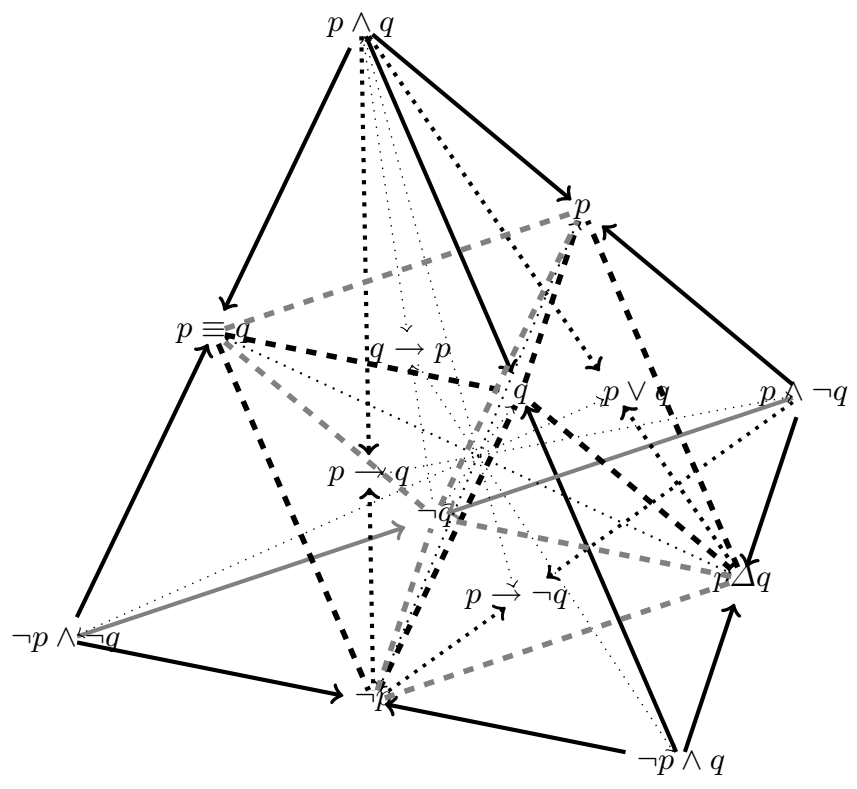

Fig. 2. The tetrahedron of the 16 binary connectives 


\section{$2.2 \quad$ Rough Sets}

In rough set theory, knowledge about objects is represented in terms of observables (attributes) collected in an Information Table (or System) [13, 14].

Definition 2.1. An Information Table is a structure $\langle O b j, A$, val, F $F$ where:

- the universe $O b j$ is a non empty set of objects;

- A is a non empty set of condition attributes;

- val, the set of all possible values that can be observed for all attributes;

- $F$ (the information map) is a mapping $F: O b j \times A \rightarrow$ val which associates to any pair object-attribute, the value $F(x, a)$ assumed by a for the object $x$.

In standard rough set theory we define an indiscernibility relation with respect to a set of attributes $B \subseteq A$ as $x \mathcal{I}_{B} y \quad$ iff $\quad \forall a \in B, F(x, a)=F(y, a)$. This is an equivalence relation, which partitions $X$ into equivalence classes $[x]_{B}$, our granules of information. Due to a lack of knowledge we are not able to distinguish objects inside the granules, thus, not all subsets of $O b j$ can be precisely characterized in terms of the available attributes $B$. However, any set $X \subseteq O b j$ can be approximated by a lower and an upper approximation, respectively defined as:

$$
L_{B}(X)=\left\{x:[x]_{B} \subseteq X\right\} \quad U_{B}(X)=\left\{x:[x]_{B} \cap X \neq \emptyset\right\}
$$

By set complementation of $O b j$ we can also define the exterior region $E_{B}(X)=$ $\left(U_{B}(X)\right)^{c}$, that is the objects surely not belonging to $X$. Finally, the difference between upper and lower approximations is named boundary and denoted as $\operatorname{Bnd}(X)=U(X) \backslash L(X)$. An important (with respect to the present work) property of approximations is the duality between lower and upper approximations: $L\left(X^{c}\right)=U^{c}(X)$ which permits to define the lower approximation given the upper one and vice versa.

Several generalizations of this approach are known in literature, which can concern the indiscernibility relation (hence, we have for instance similarity rough sets $[16,17]$ ), the subsethood relation (see the Variable Precision Rough Sets [10]) or the data under investigation which can be described by fuzzy sets instead of classical sets [8]. We will give more details in the following sections when needed.

\section{Opposition from Approximations}

The first obvious way to define oppositions in the rough set framework is to consider approximations. Indeed, we have that the lower approximation and the exterior region are disjoint sets (also, orthopairs [7]). So, given a subset of objects $X \subseteq O b j$, we have a tri-partition of the universe $O b j=L(X) \cup B n d(X) \cup E(X)$ and consequently a hexagon of oppositions.

We note that in the upper part of the hegaxon we have the sets representing certain knowledge on the universe, namely $L(X), E(X)$ and their union. On the other hand on the other half of the hegaxon we have uncertain knowledge: $U(X), U\left(X^{c}\right)$ and at the bottom the boundary region. 


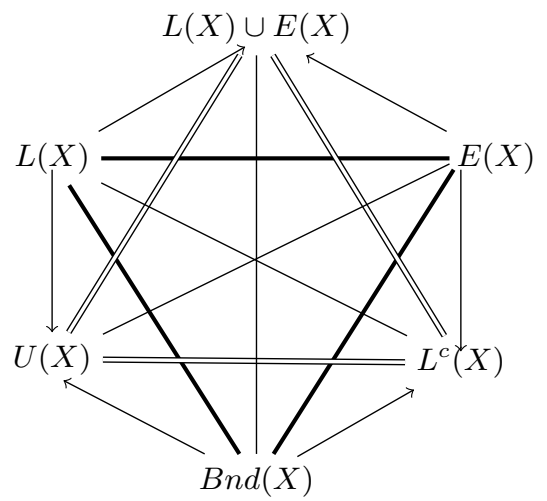

Fig. 3. Hexagon induced by Pawlak approximations

In more generalized models of rough sets, we do not have the duality between lower and upper approximations [6]. This happens, for instance, in Probabilistic Rough Sets [18] or Fuzzy Rough sets $[8,15]$. When considering opposition in this generalized framework we have a splitting in two parts of the hexagon. In figure 4 the cube of opposition resulting from this splitting is represented, where the upper and lower part are omitted. We also note that the square on the diagonals, i.e., those with vertices $\left(L(X), L\left(X^{c}\right), L^{c}(X), L^{c}\left(X^{c}\right)\right)$ and $\left(U(X), U\left(X^{c}\right), U^{c}(X), U^{c}\left(X^{c}\right)\right)$ are examples of Piaget group ${ }^{3}[9]$.

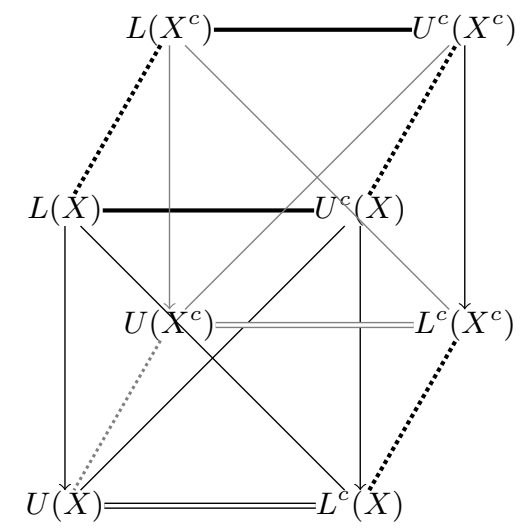

Fig. 4. Cube of opposition induced by generalized approximations

\footnotetext{
${ }^{3}$ Given a logical formula $\phi=f(p, q, r, \ldots)$, the Piaget group is the set of four transformations: identity $I(\phi)=\phi$; negation $N(\phi)=\neg \phi$; reciprocation $R(\phi)=$ $f(\neg p, \neg q, \neg r, \ldots)$ and correlation $C(\phi)=\neg f(\neg p, \neg q, \neg r, \ldots)$.
} 


\section{Opposition from Relations}

Looking at the definition of the indiscernibility relation $x R_{E} y$ iff $\forall a \in B \subseteq$ $A, F(x, a)=F(y, a)$, it can be easily seen that there other kinds of relations that can be generated, changing $\forall$ with $\exists$ and $=$ with $\neq$. Thus, given a subset of attributes $B \subseteq A$, we can define four relations:

$$
\begin{aligned}
& x R_{E} y \quad \text { iff } \quad \forall a \in B \subseteq A, F(x, a)=F(y, a) \quad \text { Indiscernibility } \\
& x R_{S} y \quad \text { iff } \quad \exists a \in B \subseteq A, F(x, a)=F(y, a) \quad \text { Partial Identity } \\
& x R_{D} y \quad \text { iff } \quad \exists a \in B \subseteq A, F(x, a) \neq F(y, a) \quad \text { Discernibility } \\
& x R_{P} y \quad \text { iff } \quad \forall a \in B \subseteq A, F(x, a) \neq F(y, a) \quad \text { Complete Difference }
\end{aligned}
$$

The discernibility relation and complete difference are the negation, respectively, of the equivalence relation and partial identity. So, it can be easily seen that these four relations form a classical square of oppositions (figure 5, left) of the AEIO kind. Moreover, this situation can be generalized considering any similarity (i.e., reflexive and symmetric) relation $[16,17]$ instead of partial identity.

Examples of a similarity relation are: two objects are similar if they have a fixed percentage of equal attributes or two objects are similar if the distance between their value of some numerical attribute is less than a fixed threshold. Then, instead of the complete difference we consider the negation of the similarity: $x R_{P} y$ iff not $x R_{S} y$, which is a preclusivity relation, i.e., a relation which is anti-reflexive and symmetric. If we characterize each relation by its required properties, we obtain the square of opposition on the right in figure 5, where $\mathrm{r}=$ reflexivity, $\mathrm{s}=$ symmetry, $\mathrm{t}=$ transitivity and $\mathrm{i}=$ irreflexivity.
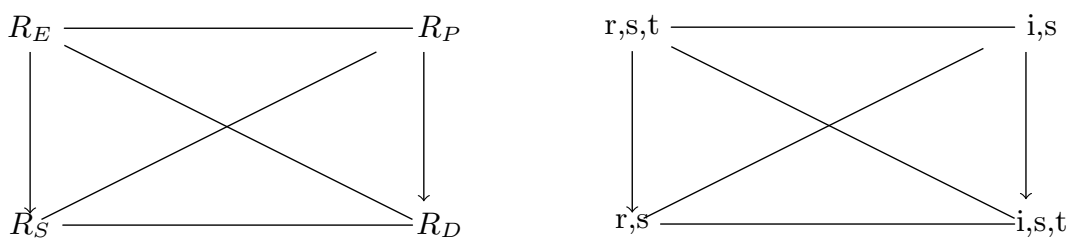

Fig. 5. Square induced by the 4 relations

Example 4.1. Let us consider a medical information table where patients are classified according to five attributes: Temperature, Pressure, Headache, Cold and Muscle-Pain. Then, two objects are equivalent if they have all the attributes equal; they are discernible if they differ for at least one attribute and we can define similarity as having three (out of five) attributes equal and consequently preclusivity is having at least three attributes different. 
As far as approximations are concerned, we notice that based on a similarity relation, lower and upper approximations can be defined as in equations 1 (other possibilites have been investigated in literature, see for instance $[12,19])$. On the other hand, given a preclusivity relation $R_{p}$, and a set of objects $X$, we can build the set of objects which are different from all the objects in $X: X^{\#}=$ $\left\{y \in O b j, \forall x \in X\left(x R_{p} y\right)\right\}$. Then, we define the upper approximation of a set as $U(X)=X^{\# \#}$ and the lower approximation is defined by duality as $L(X)=$ $\left(U\left(X^{c}\right)\right)^{c}[5]$. Discernibility relation is a special case of preclusivity, so we can proceed in the same manner. So, also with these approximations, we obtain the hexagon of Figure 3.

It is worth noticing that the approximations obtained by the discernibility relation are equal to the approximations obtained by the equivalence relations computed on the same set of attributes. That is, given a set of attributes $B$ and a set of objects $X$ we get $L_{R_{E}}^{B}(X)=L_{R_{D}}^{B}(X)$ and the same for the upper approximation. So, with respect to approximations, it is the same to ask for equivalence or discernibility of objects. On the other hand, in the case of similarity and preclusivity, given one relation we can define the other by negation but the approximations differ. In general, it holds $L_{R_{S}}^{B}(X) \subseteq L_{R_{P}}^{B}(X) \subseteq X \subseteq U_{R_{P}}^{B}(X) \subseteq U_{R_{S}}^{B}(X)$.

\subsection{Tetrahedron from Two Relations}

Let us consider two relations $R_{1}$ and $R_{2}$ of any of the four types outlined above: equivalence, similarity, discernibility and preclusivity. If we want to aggregate them, we have 14 different combinations (including $R_{1}, R_{2}$ and their negations) using the classical logic connectives. So, for instance $\left(R_{1}\right.$ AND $\left.R_{2}\right)$ requires that two objects are simultaneously related according to $R_{1}$ and to $R_{2}$. Clearly, these combinations form a tetrahedron of oppositions as described in section 2.1. Contradiction and tautology, which can be found in the middle of the tetrahedron, can here be interpreted as the fact that no objects are in the aggregated relation or that all objects are related.

Let us consider the rough set standpoint and address the problem of computing the lower and upper approximations generated by the aggregation of the two relations $R_{1} \odot R_{2}$. First of all, let us note that the effect of NOT is to turn a similarity relation into a preclusivity one (resp., equivalence into discernibility) and vice versa. Then, generally speaking, it is not possible to use directly the relation $R_{1} \odot R_{2}$ to compute approximations since it can be of none of the above four kinds.

Example 4.2. Let us consider the information table of example 4.1. Then, we define $R_{1}=$ equivalence relation on the attributes TTemperature, Cold $\}$ and $R_{2}=$ preclusivity relation consisting in having at least two (out of five) different attributes. Clearly, we are able to compute the approximations with respect to $R_{1}$ and $R_{2}$ but not with respect to the relation $R_{1}$ AND $R_{2}=$ having equal values for Temperature, Cold AND at least two different attributes.

So, the corresponding lower and upper approximations can only be obtained by computing separately the approximations obtained by the two relations and 
then aggregating them by the set operation corresponding to $\odot$. That is, we can give the following definitions

$$
\begin{array}{rr}
L_{R_{1} \mathrm{AND} R_{2}}(X):=L_{R_{1}}(X) \cap L_{R_{2}}(X) & U_{R_{1} \mathrm{AND} R_{2}}(X):=U_{R_{1}}(X) \cap U_{R_{2}}(X) \\
L_{R_{1} \mathrm{OR} R_{2}}(X):=L_{R_{1}}(X) \cup L_{R_{2}}(X) & U_{R_{1} \mathrm{OR} R_{2}}(X):=U_{R_{1}}(X) \cup U_{R_{2}}(X)
\end{array}
$$

and similarly, XOR is turned into union minus intersection, $\equiv$ in intersection plus the complement of the union.

Example 4.3. Let us consider the two relations of example 4.2 and the following information table.

Table 1. Medical information table.

\begin{tabular}{|c|ccccc|}
\hline Patient & Temperature & Pressure & Headache Cold Muscle Pain \\
\hline$p_{1}$ & high & normal & yes & yes & yes \\
$p_{2}$ & high & high & no & yes & yes \\
$p_{3}$ & normal & low & yes & no & no \\
$p_{4}$ & very high & normal & yes & yes & no \\
\hline
\end{tabular}

Let us define the set of patients $X=\left\{p_{2}, p_{3}\right\}$. Then, we have $L_{R_{1}}(X)=\left\{p_{3}\right\}$, $U_{R_{1}}(X)=\left\{p_{1}, p_{2}, p_{3}\right\}, L_{R_{2}}(X)=U_{R_{2}}(X)=\left\{p_{2}, p_{3}\right\}$. So, $L_{R_{1} \mathrm{AND} R_{2}}(X)=$ $\left\{p_{3}\right\}, U_{R_{1} \mathrm{AND} R_{2}}(X)=\left\{p_{2}, p_{3}\right\}, L_{R_{1} \mathrm{OR} R_{2}}(X)=\left\{p_{2}, p_{3}\right\}$ and $U_{R_{1} \mathrm{OR} R_{2}}(X)=$ $\left\{p_{1}, p_{2}, p_{3}\right\}$.

In this way we obtain a new tetrahedron where instead of the aggregation of relation we have the aggregation of approximations. Contradiction and tautology correspond to the emptyset and the whole universe, respectively.

Finally, we can give two interpretations to $R_{1}, R_{2}$ and their aggregation:

1. the relations represent the standpoint of two different agents with their own knowledge about the same phenomenon. Then, by aggregation of the two relations, we obtain an aggregation of their points of view. Thus, we are in a situation similar to [11] with the difference that our relations are not necessarily equivalence ones. In analogy to the work by Khan and Banerjee, the approximations obtained by AND of the two relations can be named strong approximations and the one obtain by the OR as weak approximations.

2. $R_{1}$ and $R_{2}$ correspond to a single agent having different requirements on different attributes. For instance, consider again the medical information table. In this framework, an agent can ask for instance that two patients are in relation if they have same value for the attributes $B_{1}=$ \{Headache, Muscle-Pain, Cold $\}$ and similar values for $B_{2}=\{$ Temperature, Pressure $\}$. This corresponds to search for the pair $x R_{E}^{B_{1}} y$ AND $x R_{S}^{B_{2}} y, R_{S}$ needing to be properly defined. 


\section{Conclusions}

We have analyzed rough set theory in the light of the theory of oppositions. Several kinds of opposition and their geometrical organizations can be laid bare.

New scenarios are also highlighted on the aggregation of two relations and on the possibility that a single agent expresses different requirements on the same data. One can also suppose to further generalize this approach to $n$ relations. In this case the number of possible connectives is $2^{2^{n}}$ (including contradiction and tautology). The interpretation in rough sets is a clear extension of what is said above: $n$ agents or $n$ different requirements from a single agent. On the contrary, the geometrical organization of oppositions in this setting becomes problematic, since the number of connectives would become intractable even for small values of $n$.

Finally, we note that the hexagon and the cube of Figure 3 and 4 have hexagon and cube counterparts in FCA (and possibility theory) in [9]. But as explained in [9] they are not regular hexagon and cube (indeed, some links have a different meaning) while in the rough set case the hexagon and the cube are regular ones (in the sense of Blanché). This reveals that the structure of opposition in rough sets and FCA are different, which offers a new departure point for comparing the two theories.

\section{References}

1. Béziau, J.Y., Gan-Krzywoszyńska, K. (eds.): The Square of Opposition (2010), http://www.square-of-opposition.org/Square2010-handbook.pdf

2. Béziau, J.Y., Payette, G.: Preface. Special issue on the square of opposition. Logica Universalis 2, 1-1 (2008)

3. Béziau, J.Y., Payette, G. (eds.): Handbook of the Second World Congress on the Square of Opposition. Peter Lang, Pieterlen, Switzerland (2012)

4. Blanché, R.: Sur l'opposition des concepts. Theoria 19, 89-130 (1953)

5. Cattaneo, G.: Generalized rough sets (preclusivity fuzzy-intuitionistic BZ lattices). Studia Logica 58, 47-77 (1997)

6. Ciucci, D.: Approximation algebra and framework. Fundamenta Informaticae 94(2), 147-161 (2009)

7. Ciucci, D.: Orthopairs: A simple and widely usedway to model uncertainty. Fundamenta Informaticae 108(3-4), 287-304 (2011)

8. Dubois, D., Prade, H.: Putting rough sets and fuzzy sets together. In: Słowinski, R. (ed.) Intelligent Decision Support - Handbook of Applications and Advances of the Rough Sets Theory, pp. 203-232. Kluwer Academic Publishers, Dordrecht (1992)

9. Dubois, D., Prade, H.: From Blanché hexagonal organization of concepts to formal concept analysis and possibility theory. Logica Universalis pp. 1-21 (2012), http://dx.doi.org/10.1007/s11787-011-0039-0, in press

10. Katzberg, J., Ziarko, W.: Variable precision extension of rough sets. Fundamenta Informaticae 27, 155-168 (1996)

11. Khan, M.A., Banerjee, M.: A study of multiple-source approximation systems. Transactions on Rough Sets 12, 46-75 (2010) 
12. Lin, T.Y., Huang, K.J., Liu, Q., Chen, W.: Rough sets, neighborhood systems and approximation. In: Proceedings of the Fifth International Symposium on Methodologies of Intelligent Systems, Selected Papers. pp. 130-141 (1990)

13. Pawlak, Z.: Information systems - theoretical foundations. Information Systems 6, 205-218 (1981)

14. Pawlak, Z., Skowron, A.: Rudiments of rough sets. Information Sciences 177, 3-27 (2007)

15. Radzikowska, A., Kerre, E.: A comparative study of fuzzy rough sets. Fuzzy Sets and Systems 126, 137-155 (2002)

16. Skowron, A., Stepaniuk, J.: Tolerance approximation spaces. Fundamenta Informaticae 27, 245-253 (1996)

17. Słowinski, R., Vanderpooten, D.: Similarity relation as a basis for rough approximations. In: Wang, P. (ed.) Advances in Machine Intelligence and Soft-Computing, vol.IV, pp. 17-33. Duke University Press, Durham, NC (1997)

18. Yao, J.T., Yao, Y., Ziarko, W.: Probabilistic rough sets: Approximations, decisionmakings, and applications. Int. J. Approx. Reasoning 49(2), 253-254 (2008)

19. Yao, Y.: Relational interpretations of neighborhood operators and rough set approximation operators. Information Sciences 111, 239-259 (1998) 